\title{
Consumo simbólico e representação do self: um estudo de interações em uma comunidade virtual de usuários Ubuntu-Br
}

\author{
Osíris luís da CunHa Fernandes ${ }^{1}$ \\ Nelson da Cruz MONTEIRO FERNANDES ${ }^{2}$ \\ FERNANDO GOMES dE PAIVA JÚNIOR ${ }^{3}$ \\ ANdRÉ LUIZ MARANHÃo de SOUZA LEÃO ${ }^{3}$ \\ MARCONI FREITAS DA COSTA ${ }^{2}$
}

\author{
1 Instituto de Tecnologia de Pernambuco (ITEP), ReCife - PE, Brasil \\ 2 Universidade Federal de Pernambuco (UFPE) / Núcleo de Gestão, CARUaru - PE, BRASIL \\ 3 Universidade Federal de Pernambuco (UFPE) / Departamento de CiênCIAS AdMINISTRATIVAS, ReCIFE - PE, BRASIL
}

\begin{abstract}
Resumo
Este estudo tem como objetivo explicar como o consumo simbólico do sistema operacional Ubuntu é utilizado para a representação do self em interações na comunidade virtual Ubuntu do Brasil. Adotamos a concepção Goffmaniana do self, a netnografia da comunicação como método de pesquisa e o estudo de caso como estratégia de pesquisa. O paralinguístic, o extralinguístico e a definição do "eu" são aspectos usados nas interações virtuais. Eles têm a função linguística de corroborar e elogiar as declarações de migração de usuários do Windows para o Ubuntu, enfatizando as características distintivas do Ubuntu, destacando a expressão de sentimentos compartilhados de amor e liberdade, como formas de projetar o self de humanidade entre si. No caso do sistema operacional, essa característica é representada através da provisão de suporte entre usuários no fórum da comunidade virtual.
\end{abstract}

Palavras-chave: Consumo Simbólico. Self. Netnografia da Comunicação. Comunidades Virtuais. Ubuntu.

Symbolic consumption and representation of self: a study of interactions in a virtual community of Ubuntu-Br users

\section{Abstract}

This study aims to explain how the symbolic consumption of the Ubuntu operating system is used for the representation of self in interactions in the Ubuntu virtual community from Brazil. We adopted the Goffmanian concept of self, the netnography of communication as the research method, and case study as a research strategy. The paralinguistic, the extralinguistic, and the definition of "I" are aspects used in virtual interactions. They have the linguistic function of corroborating and praising the statements of migration of Windows users to Ubuntu, emphasizing the distinctive features of the concept of Ubuntu, highlighting its expression of shared feelings of love and freedom, as ways of projecting the self of humanity to each other. In the case of the operating system, this characteristic is represented through the provision of support among users at the forum of the virtual community.

Keywords: Symbolic Consumption. Self. Netnography of Communication. Virtual Communities. Ubuntu.

\section{El consumo simbólico y la representación del self: un estudio de las interacciones en una comunidad virtual de usuarios Ubuntu-Br}

\section{Resumen}

Este estudio tiene como objetivo explicar cómo el consumo simbólico del sistema operativo Ubuntu es utilizado para la representación del self en interacciones en la comunidad virtual Ubuntu de Brasil. Adoptamos la concepción goffmaniana del self, la netnografía de la comunicación como método de investigación y el estudio de caso como estrategia de investigación. El paralingüístico, el extralingüístico y la definición del self son aspectos usados en las interacciones virtuales. Ellos tienen la función lingüística de corroborar y elogiar las declaraciones de migración de usuarios de Windows a Ubuntu, enfatizando las características distintivas de Ubuntu, destacando la expresión de sentimientos compartidos de amor y libertad como formas de proyectar el self de humanidad entre sí. En el caso del sistema operativo, esta característica se representa a través de la provisión de soporte entre usuarios en el foro de la comunidad virtual.

Palabras clave: Consumo simbólico. Self. Netnografía de la comunicación. Comunidades virtuales. Ubuntu. 
Consumo simbólico e representação do self: um estudo de interações em uma comunidade virtual de usuários Ubuntu-Br
Osíris Luís da Cunha Fernandes | Nelson da Cruz Monteiro Fernandes Fernando Gomes de Paiva Júnior | André Luiz Maranhão de Souza Leão Marconi Freitas da Costa

\section{INTRODUÇÃO}

A integração da informação em redes globais tem causado a superação do Estado-nação neste tempo marcado pela globalização, especialmente em áreas econômica, cultural e política (GUAN, WANG, JIN et al., 2018). Por outro lado, o capitalismo transformado pelo informacionalismo e a vigilância constante por meio da web (HIMANEN, 2001; MCCAHILL, 2002) são duas das características mais marcantes deste tempo e têm dificultado o controle das atividades econômicas no interior das fronteiras nacionais (MORGAN, 2001).

Nesse contexto, as atividades de consumo permeiam a interação entre as pessoas (CHAN e LI, 2010), e não mais exigem a presença física, uma vez que pode ocorrer de forma virtual ou digital (CASTELLS, 2002; HABIBI, LAROCHE e RICHARD, 2016). Da mesma forma, bens são usados como valor de referência de um ideal; representam um significado atribuído a objetos materiais e simbólicos (SCHOUTEN, 1991; BANISTER, 2004). Em outras palavras, os indivíduos não mais compram produtos ou serviços tão somente em função de suas funcionalidades, mas também em busca do valor simbólico ao qual desejam se associar (RAVASI e RINDOVA, 2008).

No caso do consumo on-line, muito do valor relacionado à função ou uso de um produto torna-se invisível (WITT, 2010), uma vez que a percepção desse valor parece atrelada a elementos simbólicos a ele associados (LUNA-CORTES, 2017). Portanto, os atos de compra e consumo também representam esforços proativos de posicionamento do consumidor no sistema de relações sociais (SCHAU e GILLY, 2003), considerando que a mudança de características dos artefatos leva a que sejam utilizados como representações simbólicas, na medida em que refletem, expressam e reconstituem o sistema de relações sociais dos consumidores (HAMMERL, DORNER, FOSCHT et al., 2016).

Assim, quando pesquisamos o consumo simbólico do Sistema Operacional (SO) de base Linux, denominado Ubuntu, não podemos analisá-lo considerando apenas as características funcionais, mas, fundamentalmente, os significados que ele pode articular. Para tanto, neste estudo, abraçamos a perspectiva teórica da Sociolinguística Interacional (GUMPERZ, 1999) que prioriza a análise contextual, e permite acessar os interagentes da comunidade virtual Ubuntu como potenciais fontes de comunicação e cujas ações e intenções de significação, comportamentos verbais e não-verbais, pretendemos entender em seu contexto imediato. A sociolinguística nos permite investigar o uso da linguagem pelos membros dessa comunidade, dando conta da junção entre os aspectos paralinguísticos e sociais com respeito a seu processo de comunicação (GUMPERZ, 1982; SAVILLE-TROIKE, 2003).

Neste estudo, lidamos com a representação simbólica a partir do uso da concepção de self desenvolvido sob a ótica da teoria social de Erwin Goffman (1990), cujas raízes se encontram no interacionismo simbólico, como forma de nos aproximarmos da compreensão da representação do self em interações online por meio do consumo simbólico do Sistema Operacional Ubuntu. O estudo objetiva responder a seguinte questão de pesquisa: como ocorre a representação do self em interações virtuais por meio do consumo simbólico do Sistema Operacional Ubuntu?

\section{A comunidade virtual dos usuários do Ubuntu}

A história do sistema operacional Ubuntu deve ser contada dentro do espectro mais amplo do movimento de software livre no Brasil e no mundo, e seu antagonismo com outras lógicas de produção e consumo de software (LOWE, POW, SERBAN et al., 2017), como o sistema Microsoft e outros modelos corporativos.

No Brasil, o Ubuntu-BR é a materialização desse movimento e segue a mesma lógica adotada em outros países. A iniciativa brasileira utiliza o Manifesto Ubuntu mundial como base para que os usuários aproveitem o software gratuitamente, traduzido livremente para o português e com a possibilidade de adaptar o código de modo a ser inclusivo a pessoas com deficiência.

O Ubuntu revela seu alinhamento com a filosofia do software livre, baseando-se em quatro conceitos básicos de liberdade: uso, cópia, modificação e redistribuição (SILVEIRA, 2004). O usuário pode usar legalmente o sistema operacional Ubuntu, fazer alterações e adaptações, além de fazer cópias e redistribuir informações livremente. Esses conceitos permitem a acumulação de conhecimento por meio de contribuições bem-vindas, feitas por qualquer usuário.

Para assegurar que esses quatro "princípios de liberdade" fossem mantidos, o criador do conceito de software livre, Stallman (2002), projetou a Licença Pública Geral. Esta licença visa impedir a introdução de barreiras ao uso de software livre, desde que sejam mantidos os preceitos básicos da criação (SODERGERG, 2012). Além disso, o fato de o sistema operacional ser 
Consumo simbólico e representação do self: um estudo de interações em uma comunidade virtual de usuários Ubuntu-Br
Osíris Luís da Cunha Fernandes | Nelson da Cruz Monteiro Fernandes Fernando Gomes de Paiva Júnior | André Luiz Maranhão de Souza Leão Marconi Freitas da Costa

um software e, portanto, imaterial, permite que ele seja consumido simultaneamente por várias pessoas. Segundo Lessig (2005), essa exigência de não-rivalidade e não-exclusividade, típica de bens intangíveis, permite que informação, cultura e conhecimento não tenham propriedade exclusiva para satisfazer o desejo de criar e aprender. Neste contexto, a história do software livre e do Ubuntu está profundamente enraizada na participação de comunidades efetivas. A criação de uma distribuição GNU / Linux pelo Ubuntu representa o esforço para criar um modelo de ecossistema com pessoas interagindo como uma comunidade.

O Ubuntu não é o primeiro sistema operacional baseado em Linux a ser lançado, mas foi o primeiro a trazer recursos para o usuário comum, como a instalação automática de programas, pacotes de escritório (Word, Excel e Power Point da Microsoft), assim como outros recursos de configuração do sistema (VELLOZO, 2012). Como Software Livre e de Código Aberto (FLOSS), o Ubuntu é formado por um grupo dedicado de centenas de pessoas trabalhando para produzir um novo lançamento a cada seis meses. O sistema é o resultado de uma rede, executando desde o sistema operacional Debian até milhares de grandes e pequenos projetos que produzem código e documentação.

Além disso, o Ubuntu reconhece as contribuições de vários indivíduos - entusiastas, artistas, moderadores de fórum, operadores de canal IRC, organizadores de eventos comunitários, escritores, tradutores, repórteres de erros e os classificadores - qualquer que seja o talento ou interesse particular. A partir disso, o Ubuntu tem mudado o universo dos sistemas operacionais e o FLOSS, ao se tornar um sistema completo para milhões de computadores e servidores. Milhares desses usuários retornam à comunidade Ubuntu criando documentação, tradução e programação.

Para manter um fluxo satisfatório de contribuições para a marca, o Ubuntu trabalha para equilibrar a importância do papel da Canonical em empregar alguns indivíduos na comunidade. 0 projeto é baseado em uma crença fundamental de que o software é construído, apoiado e mantido apenas através de fortes relações entre os indivíduos que usam a marca. Assim, ao incentivar e apoiar essa comunidade, o sistema operacional Ubuntu obtém ganhos maiores que o modelo que valoriza apenas o desenvolvimento pago.

Para Hill e Bacon (2008), os interlocutores do projeto acreditam que a Canonical Ltda. foi a principal catalisadora do processo que construiu o Ubuntu, e a comunidade de usuários foi responsável pela sua distribuição bem-sucedida. Além disso, os líderes do projeto acreditam que o sucesso da marca só continuará a crescer por meio de uma aliança - em constante expansão com esses usuários que atuam em conformidade com a comunidade.

\section{Consumo Produtivo}

O contexto de consumo abordado é baseado no conceito de "prossumidor", que é baseado na combinação dos significados de produtor e consumidor (TOFFLER, 1980). Pieske e Prado (2012) reconhecem que o consumidor que produz seus produtos e serviços não é exatamente novo (compras de supermercado, moda "faça você mesmo", movimentos de autoajuda e associações, restaurantes de autoatendimento).

A discussão em torno dessa prática começa com um desafio de Kotler (1986) com relação à validade e extensão da tese de Toffler (1980), da existência de uma nova modalidade de consumo. Os prossumidores se engajam direta e indiretamente na criação e compartilhamento de soluções na produção de bens e serviços, reforçando a cultura de participação ativa que está em oposição à lógica do consumidor alienado-passivo (RITZER, 2015). O fenômeno do prossumo ocorreu tanto na era prémoderna quanto na era moderna (IZVERCIANU, SERAN e BRANEA, 2014) e, atualmente, a contribuição das tecnologias da informação e comunicação (TICS) para as redes, possibilitaram as habilidades produtivas desses agentes e os meios para a distribuição, captura, recuperação e organização de processos produtivos específicos.

No contexto da Web 2.0, o prossumidor é qualificado como voluntarioso e não busca lucro, mesmo quando os esforços resultam em produtos ou processos (RITZER e JUGERSON, 2010; RITZER, 2014). No contexto das tecnologias da Web 2.0, a chegada de bens e serviços a novos mercados é potencializada pelas interações virtuais de prossumidores (VAN DIJCK, 2009; ALMEIDA, MAZZON, MULLER NETO et al., 2012).

O comportamento desse prossumidor significa uma forma de empoderamento, capacitando o consumidor como força política e agente de transformação (CEVASCO, 2008). O prossumo forma um espaço para a reprodução das forças de mercado e um lugar de produção de significados. Assim, pode ser um espaço de luta que não se restringe à posse de objetos, pois a ênfase está no seu uso, que é o que dá aos objetos uma forma social. 
Consumo simbólico e representação do self: um estudo de interações em uma comunidade virtual de usuários Ubuntu-Br
Osíris Luís da Cunha Fernandes | Nelson da Cruz Monteiro Fernandes Fernando Gomes de Paiva Júnior | André Luiz Maranhão de Souza Leão Marconi Freitas da Costa

Algumas obras contemporâneas continuam a se referir ao trabalho seminal de Kotler, destacando sua importância. Esses estudos, no entanto, não mostram avanços teóricos significativos e apresentam uma predominância do debate em torno dos conceitos e da abordagem gerencial / utilitarista. Fonseca, Gonçalves, Oliveira et al. (2008) abordam o prossumidor como um "novo usuário" que produz e consome os produtos e serviços que "precisam", tecendo um perfil de um super indivíduo com "novas habilidades". Esta condição permite ao prossumidor se diferenciar do consumidor "normal" que está fora da realidade da Web 2.0. Tal abordagem levanta uma leitura histórica sem explorar as transformações e forças culturais mais amplas que ditam a direção do mercado (COMOR, 2011).

\section{Consumo Simbólico}

Os behavioristas utilizam o termo consumo simbólico para descrever atos de compra e consumo de produtos para fins sociais e de auto expressão, definidos como a comunicação da identidade social e individual (RAVASI e RINDOVA, 2008; DEANS, THOMAS, DAUBE et al., 2016). Mais recentemente, mudanças nos valores culturais e padrões de consumo nos fazem repensar o papel dos objetos na vida das pessoas em sociedades afluentes (TANGSUPWATTANA e LIU, 2017). Assim, em sua produção, os objetos têm passado por reformulações em forma, aparência, cor, estilo e sentimento, mudando a maneira como os consumidores os experienciam e as associações que eles evocam (SHANG, CHEN e HUANG, 2012).

Nessa perspectiva, os objetos deixam de ser vistos apenas como ferramentas funcionais e passam a ser valorizados pelos significados a eles atribuídos em um determinado contexto cultural (PRONAY e HETESI, 2016). Ou seja, os objetos devem ser comprados e usados não apenas por sua capacidade de desempenhar funções específicas, mas, pelos seus significados e pelo desejo de possuí-los para mostrar aos outros. Os objetos servem, assim, o propósito de ratificar status e a identidade individual ou coletiva (RAVASI e RINDONA, 2008; GBADAMOSI, 2015).

As mudanças nos padrões de consumo e valores culturais promoveram o surgimento de novas expectativas e objetivos em torno do papel dos bens tecnológicos na vida das pessoas (HAMARI e KERONEN, 2017). Os bens são símbolos, cuja posse e uso refletem, expressam e reconstituem o sistema de relações sociais e seu lugar percebido (EKINCI, SIRAKAYA-TURK e PRECIADO, 2013). Tais mudanças, entretanto, têm um impacto direto nas questões relacionadas à identidade (SCHOUTEN, 1991), entendidas como o processo de construção de significado baseado em um atributo cultural, ou um conjunto de atributos culturais inter-relacionados que prevalecem sobre outras fontes de significados (CASTELLS, 2010).

\section{Noções de Self na Teoria Social de Erving Goffman}

Em seus estudos, Goffman (1990) explora as particularidades da identidade individual e social e as relações em grupo numa esfera micro sociológica, por meio da observância da interação social em ações cotidianas e direcionando sua atenção para a forma como cada pessoa desempenha seu papel e gerencia as impressões que causam nos outros em diferentes contextos. Para tal, a interação social se baseia em uma metáfora teatral da vida social, segundo a qual o papel assumido por um ator social (pessoa) é estabelecido em resposta à interpretação de outros atores em cena (contexto), para um público de potenciais participantes e a impressão que o ator pretende retratar (MUELLER, 2018).

Para Blumer (1986), o termo "interação simbólica" refere-se à natureza peculiar e distinta da interação entre os seres humanos. A peculiaridade consiste no fato de que os seres humanos interpretam ou definem as ações uns dos outros, em vez de simplesmente reagirem (DIONYSIOU e TSOUKAS, 2013). Assim, a interação humana é mediada pelo uso e interpretação de símbolos ou pela determinação do significado de cada um na reação (KOTARBA, 2014).

$\mathrm{Na}$ abordagem interacionista simbólica, as pessoas devem interpretar o significado das coisas, eventos e ações que não são originados na relação com o mundo, mas emergem da interação social (HAMMERL, DORNER, FOSCHT et al., 2016). Portanto, sob essa ótica, os símbolos e seus significados desempenham um papel essencial na formação do self, uma vez que constituem elementos exclusivamente humanos e sociais, embora possam surgir da relação entre esses componentes, bem como modificar e ser modificados por eles nessa relação (GRUBB e GRATHWOHL, 1967; BAUMHAMMER, SILVA e COSTA, 2017). Isso significa que não há relação de passividade entre o indivíduo e a sociedade (McCRACKEN, 1990). A figura 1 apresenta um resumo das principais características da abordagem do interacionismo simbólico.

As teorias do self que compõem a tradição interacionista simbólica são originárias da concepção de James (1980), que diferencia os "aspectos discriminados" do self, tendo-os designado como Eu e Mim (GECAS, 1982). Para ele, nenhum aspecto pode ser analisado adequadamente sem que o outro tenha sido considerado (HARMON-KIZER, KUMAR, ORTINAU et al., 2013). É por isso que o "Eu" corresponde ao aspecto "puro" do self, ao "sujeito", a um senso de identidade pessoal. 
Consumo simbólico e representação do self: um estudo de interações em uma comunidade virtual de usuários Ubuntu-Br
Osíris Luís da Cunha Fernandes | Nelson da Cruz Monteiro Fernandes Fernando Gomes de Paiva Júnior | André Luiz Maranhão de Souza Leão Marconi Freitas da Costa

Figura 1

Características principais do interacionaismo simbólico

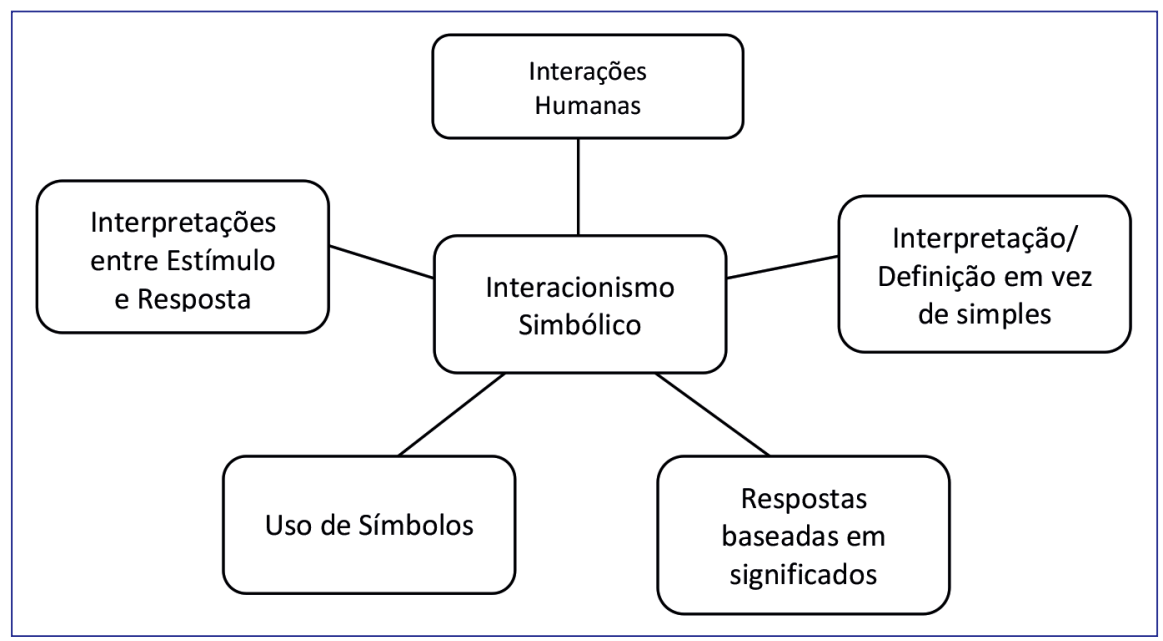

Fonte: Fonte: Elaborado pelos autores.

A perspectiva interacionista-simbólica de Goffman reflete sua busca por compreender as microestruturas sociais por meio da interação. Para Mead (2015), a sociedade é um tecido de comunicação no qual as pessoas, por meio da interação, se influenciam mutuamente, o que torna o self e a sociedade mutuamente constituídos por meio da linguagem. Essa compreensão da linguagem é estabelecida em seu efeito prático, em que o significado sempre vem de seus contextos de uso, uma noção teorizada inicialmente por Wittgenstein (2003). Assim, segundo Habermas (2002), justamente porque as interações sociais funcionam como jogos de linguagem que é possível ter um desenvolvimento simultâneo entre a constituição do self e a estrutura social.

O conceito de jogo de linguagem de Wittgenstein consegue traduzir bem a multiplicidade de práticas e mudanças pragmáticas destinadas a persuadir os atores sociais a ampliar o alcance de seus compromissos com os outros e as táticas usadas na construção de comunidades inclusivas, como a Comunidade Virtual de Usuários Ubuntu. Segundo Goffman (1990), isso confirma a tese de que o humano é socialmente construído a partir de sua alteridade. Ou seja, sua identidade é construída a partir da interação com os pares, cada um em seu papel social, visando conhecer a si e aos outros em interação.

Além disso, os jogos de linguagem usados na costura dessas redes são feitos por regras tácitas que exigem acordos intersubjetivos existentes na sociedade antes que qualquer processo possa funcionar. Isso porque, de acordo com Wittgenstein (2003), acordos sociais não dependem apenas de regras; deve haver um acordo sobre como eles são usados. A compreensão através da linguagem não é apenas uma concordância nas definições, mas também (por mais estranho que possa parecer) uma concordância nos julgamentos.

Para Wittgenstein, "obedecer a uma regra" é uma prática, e nossa compreensão das regras consiste no domínio de uma técnica. Assim, Wittgenstein entende as regras como práticas, inseparáveis de um modo de vida específico. Segundo Goffman (1990), as comunidades ajudam a construir o "eu", que se baseia nos papéis expressos nesses ambientes colaborativos, impregnados com os símbolos intermediários da comunicação e da linguagem, verbais e não verbais, bem como a concepção de si mesmos através da alteridade.

\section{PROCEDIMENTOS METODOLÓGICOS}

Esta pesquisa está em consonância com os princípios conceituais da sociolinguística interacional, que incorpora a compreensão da constituição do self através das interações sociais, fornecendo um marco para a compreensão de como os indivíduos se comportam nessas interações (GOFFMAN, 1974; GUMPERZ, 1972). Por uma questão de consistência epistemológica, está alinhado com a perspectiva pragmática da linguagem, cuja concepção permite entender que o significado dos signos pode variar dependendo de como são usados, levando para o mesmo termo que assume diferentes significados e até alguns novos ao longo do tempo (WITTGENSTEIN, 2003). Portanto, este estudo enquadra-se na tradição interpretativa que compreende a linguagem como base para a construção da realidade (GUMPERZ e HYMES, 1972), a partir de uma perspectiva interacionista simbólica (BLUMER, 1986). 
Consumo simbólico e representação do self: um estudo de interações em uma comunidade virtual de usuários Ubuntu-Br
Osíris Luís da Cunha Fernandes | Nelson da Cruz Monteiro Fernandes Fernando Gomes de Paiva Júnior | André Luiz Maranhão de Souza Leão Marconi Freitas da Costa

Quando as interações em uma comunidade online, permeadas pela dimensão simbólica do consumo do Ubuntu, são definidas como objeto de pesquisa, é possível dizer que o software é um signo socialmente compartilhado. Desse ponto de vista, o significado do Ubuntu é estabelecido ou alterado por seus usuários/consumidores na medida em que eles usam o Ubuntu como uma representação do self (ou selves) em suas interações na comunidade Ubuntu.

A comunidade e os textos utilizados para construir esta pesquisa foram escolhidos por sua natureza introspectiva, determinada pelo conhecimento que um falante da comunidade ou cultura tem da língua adotada. Uma abordagem interpretativa foi utilizada para ampliar o escopo da pesquisa, para melhor contemplar as considerações contextuais disponíveis na comunidade virtual (TRAUTH e JESSUP, 2000). O estudo é realizado com base no pressuposto de Wittgenstein (2003) (em sua segunda filosofia) de que os membros da comunidade devem compartilhar o mesmo "jogo de linguagem", entendido aqui como a linguagem entre palavras e o mundo, nas duas direções, dando sentido à relação entre realidade e objeto, caracterizando as competências dos membros para se juntarem a esse grupo. Assim, considerando nosso objetivo de entender certos aspectos culturais da comunidade on-line do Ubuntu através da interação que ocorre entre os membros, e o entendimento de que eles compartilham o modo como os signos da linguagem são usados, linguisticamente ou não (GUMPERZ, 1972), o estudo adota a netnografia como método para conduzir a pesquisa (KOZINETS, 2002; JANSEN, SPINK e TAKSA, 2009; BOWLER, 2010).

A pesquisa também adota alguns preceitos da etnografia da comunicação (SAVILLE-TROIKE, 2003), baseada tanto na linguística quanto na antropologia. O método considera a comunicação como um meio de dar sentido ao mundo, sendo parte integrante da cultura (FARAH, 1997). A comunicação é condicionada pela cultura, ao mesmo tempo em que a revela e apoia (POLUKHINA e STRELNIKOVA, 2015). Para acessar e interpretar a linguagem verbal e não verbal utilizada nas interações da comunidade Ubuntu utilizou-se o protocolo de análise proposto por Freitas e Leão (2012), por apresentar critérios desenvolvidos para a etnografia da comunicação em um contexto online. É apresentado na Quadro 1 abaixo.

\section{Quadro 1}

\section{Protocolo de análise dos dados}

\begin{tabular}{|c|c|c|}
\hline \multirow{6}{*}{ 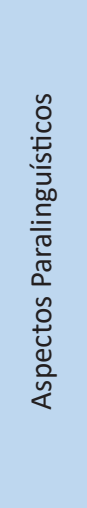 } & Acentuação & $\begin{array}{l}\text { Trata-se de alterações na escrita das palavras com acentos tônicos, de modo a reduzir a quantidade } \\
\text { de caracteres ou símbolos digitados necessários à construção da palavra, segundo a norma culta. }\end{array}$ \\
\hline & Altura da voz & $\begin{array}{l}\text { Na comunicação de internet se caracteriza, principalmente, pelo uso de todas as letras da(s) } \\
\text { palavra(s) em maiúsculo. }\end{array}$ \\
\hline & $\begin{array}{l}\text { Duração da } \\
\text { elocução }\end{array}$ & $\begin{array}{l}\text { Refere-se à repetição de letras ou uso de sinas de reticências no sentido de alongar a pronúncia } \\
\text { da palavra ou expressão. }\end{array}$ \\
\hline & Entoação & $\begin{array}{l}\text { Caracteriza-se pela utilização dos sinais de exclamação, interrogação, no sentido de demonstrar } \\
\text { surpresa, dúvida, espanto e afins. }\end{array}$ \\
\hline & Tom & $\begin{array}{l}\text { Refere-se a expressões, que não são faciais, ligadas ao tom de voz, geralmente no sentido de } \\
\text { suspirar, opinar, gabar-se, etc. }\end{array}$ \\
\hline & $\begin{array}{l}\text { Variações } \\
\text { ortoépicas }\end{array}$ & $\begin{array}{l}\text { Refere-se a mudanças na escrita das palavras e expressões, com intuito de reduzi-las, em termos } \\
\text { de tamanho, e de destacar sotaques. }\end{array}$ \\
\hline 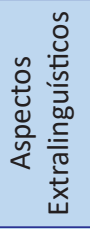 & $\begin{array}{l}\text { Expressão } \\
\text { facial }\end{array}$ & $\begin{array}{l}\text { Trata-se de palavras e símbolos escritos de modo a demonstrar expressões faciais, tais como risos, } \\
\text { gargalhadas e outros, a partir de repetições de letras ou outros usos de símbolos de uma forma } \\
\text { não-habitual (: P), por exemplo. }\end{array}$ \\
\hline $\bar{\partial}$ & $\begin{array}{l}\text { Movimento } \\
\text { dêitico }\end{array}$ & $\begin{array}{l}\text { Referem-se a expressões escritas usadas no sentido de demonstrar gestos específicos, que } \\
\text { indiquem direção, por exemplo. }\end{array}$ \\
\hline $\begin{array}{l}\frac{0}{0} \\
0 \\
\frac{2 \pi}{2} \\
\stackrel{20}{=}\end{array}$ & $\begin{array}{l}\text { Alternância } \\
\text { de código }\end{array}$ & $\begin{array}{l}\text { Ocorre quando a forma escrita de alguma palavra ou o uso de aspas nesta muda o seu sentido } \\
\text { habitual, incluindo a substituição de palavras por números, por exemplo, cujo uso específico seja } \\
\text { compreendido por um grupo cultural ou tribo. }\end{array}$ \\
\hline $\begin{array}{l}0 \\
0 \\
0 \\
\tilde{0}\end{array}$ & $\begin{array}{l}\text { Conhecimento } \\
\text { de mundo }\end{array}$ & $\begin{array}{l}\text { Caracteriza-se pelo conhecimento construído por um grupo ou pelo senso comum, demonstrado, } \\
\text { aqui, a partir do uso de palavras escritas de forma distinta da ditada pela norma culta, ou alterando } \\
\text { seu sentido habitual para outro compartilhado por um grupo, cultura ou senso comum. }\end{array}$ \\
\hline$\frac{\mathscr{0}}{\dot{\alpha}}$ & Footing & $\begin{array}{l}\text { Ocorre pelo uso de símbolos ou sinais, por exemplo, parênteses, que demarquem mudança no } \\
\text { alinhamento dos interactantes. }\end{array}$ \\
\hline
\end{tabular}

Fonte: Freitas e Leão (2012). 
Consumo simbólico e representação do self: um estudo de interações em uma comunidade virtual de usuários Ubuntu-Br
Osíris Luís da Cunha Fernandes | Nelson da Cruz Monteiro Fernandes Fernando Gomes de Paiva Júnior | André Luiz Maranhão de Souza Leão Marconi Freitas da Costa

O levantamento netnográfico foi realizado durante 12 meses, entre outubro de 2011 e outubro de 2012 e ofereceu 89 observações. A coleta de dados foi realizada por meio da compilação de depoimentos (LINCOLN e GUBA, 1985) retirados do canal "espaço de ..." no fórum da comunidade, onde a viabilidade e conveniência digital não exigiam o uso de gravador ou anotações durante a coleta, como defendido nos procedimentos usuais e etnográficos (KEATING, 2001). Os dados foram gerenciados separando os depoimentos de acordo com a data de postagem (de acordo com o período estabelecido para a pesquisa), guardando-os em pastas de arquivo virtual com os títulos dos depoimentos e classificando-os com a letra " $D$ " e data em que foram postados. Dentro das pastas de cada depoimento, foi salva a imagem da tela do computador, denominada " $\mathrm{T}$ ". Finalmente, comentários dos membros, denominados " $\mathrm{C}$ ". Assim, durante a análise dos dados, cada parte da coleção foi analisada sob o formulário D1T1C1, por exemplo, o número a seguir às letras corresponde ao número atribuído a cada instrução destacada (D), tela (T) e comentário (C).

Observando uma condição etnográfica de imersão na comunidade Ubuntu para validar a pesquisa qualitativa, nós nos juntamos à comunidade como observadores, coletando dados através da observação não participativa e seguindo a comunidade a distância. Além disso, como critério de validade e confiabilidade dos dados, utilizou-se a triangulação e a descrição rica, como preconizam Paiva Junior, Leão e Mello (2011). Além disso, seguimos as recomendações oferecidas por Alvesson e Skoldberg (2009) relacionadas à reflexividade como critério de confiabilidade (para antes e depois do evento), que gera a transformação do pesquisador. Assim, a reflexividade foi uma forma constante de contornar os elementos limitantes e potencialmente restritivos da qualidade dos dados e análises.

Para esta pesquisa, aspectos de confiabilidade e validade foram utilizados para avaliar a qualidade do estudo qualitativo, embora esses aspectos estivessem relacionados a uma abordagem positivista e a termos orientados mais para a pesquisa (KLEIN e MYERS, 1999). No entanto, segundo Pozzebon (2004), alguns autores defendem outros critérios para avaliar a qualidade da pesquisa, como (a) critério de conformidade, que se refere ao grau em que os achados da pesquisa são determinados pelos respondentes e pelas condições da pesquisa, e não pelos vieses, motivações, interesses ou perspectivas do pesquisador (BRINK, 2009); (b) transferibilidade, que se refere à obrigação dos pesquisadores de apresentar suas descobertas de forma a permitir que os leitores considerem a possibilidade de sua transferibilidade, e a avaliação da relevância dos achados relatados cabe ao leitor (ENGWARD e DAVIS, 2015); (c) plausibilidade, referindo-se à capacidade do texto de se conectar à visão de mundo do leitor e aborda as estratégias retóricas usadas para compor um texto que posiciona o trabalho como relevante para as preocupações do público-alvo (SCHULTZE, 2000); (d) autenticidade, que significa ser genuíno com a experiência de campo como resultado de "estar lá" (GOLDEN-BIDDLE e LOCKE, 1993). Atender a esses critérios garantem que o pesquisador esteve presente (ALVESSON e SKOLDBERG, 2009), e foi genuíno com a experiência de redigir o relatório; e (e) reflexividade, que implica reflexão sobre a produção de textos e uso da linguagem, e revela um tipo de consciência acerca da ambiguidade da linguagem (ALVESSON e SKOLDBERG, 2009).

\section{RESULTADOS}

As reflexões baseadas na análise do corpus formado a partir dos dados coletados na comunidade Ubuntu-Br permitiram estabelecer a existência de três aspectos relacionados aos usos e funções da linguagem que merecem destaque, uma vez que fornecem possíveis respostas à questão central do estudo, que vamos tentar responder nas considerações finais.

\section{Forjando a representação do self a partir das declarações de migração do Windows para o Ubuntu}

A declaração dos usuários do sistema operacional Windows sobre sua migração para o Ubuntu constitui uma das formas de representação do self presentes nas interações virtuais na Comunidade Ubuntu-Br. Essas interações utilizam aspectos paralinguísticos e de definição do "eu" com o objetivo de projetar, corroborar e elevar valores associados ao Ubuntu e voltado para o reconhecimento de novos usuários na comunidade identitária. O exemplo a seguir ilustra uma situação de interação entre iniciantes no uso do Ubuntu, em que um dos participantes afirma entusiasticamente para o grupo: 
Consumo simbólico e representação do self: um estudo de interações em uma comunidade virtual de usuários Ubuntu-Br
Osíris Luís da Cunha Fernandes | Nelson da Cruz Monteiro Fernandes Fernando Gomes de Paiva Júnior | André Luiz Maranhão de Souza Leão

[Ubunteros, bom dia e bom domingo!!

Depois de um mês somente no live-CD e duas semanas só utilizando Ubuntu ( 2 instalações rsrs) posso dizer que há uma semana não vejo o outro sistema operacional que quase obrigatoriamente recebemos ( $\mathrm{\$} 500$ ou mais "despercebidos") nos PCs em geral].

O exemplo mostra a situação de uma pessoa que migrou recentemente do Windows para o Ubuntu. Essa migração fica clara pelo uso de aspectos de definição do "eu" [Alternância de código: 'live-CD'; PCs; R\$500; Footing: '(2 instalações rsrs)'; '(R\$500 ou mais "despercebidos")'; conhecimento de mundo: 'live-CD'; 'PC'], aspectos paralinguísticos (entoação: '!!') e aspectos extralinguísticos (expressão facial: rsrs) no sentido de se projetar na comunidade Ubuntu-Br, mostrando que conhece "o outro sistema operacional" (O Windows), mas que optou pelo Ubuntu e quer ser reconhecido como um usuário desse sistema.

O usuário começa demonstrando conhecimento do mundo, chamando outros interagentes de 'Ubunteros', o que também constitui uma tentativa de quebrar o gelo (reforçada pela saudação cortês, 'Bom dia e espero que todos estejam tendo um bom domingo !!]') Entendemos que o uso da entoação ('!!') no final da primeira frase é um aviso aos outros membros, a fim de ser reconhecido como uma pessoa alegre/simpática e educada (com uso de expressão facial 'rsrs', representando como risos/demonstração de alegria).

A palavra "despercebido" entre aspas (uso: footing nessa situação) pode ser entendida como uma crítica aos usuários do Windows que não se importam um com o outro, mas também pode significar a demarcação de uma posição condizente com o usuário típico do Ubuntu, que não deixa as pessoas em dificuldades à sua mercê (ou passarem despercebidas).

Estudiosos como Ravasi e Rindova (2008) argumentam que os significados sociais dos produtos atuam na dimensão dos desejos pessoais e estão relacionados à busca de diferenciação social. Consciente ou inconscientemente, os consumidores estão tomando suas decisões de compra inspiradas em sua identidade ou na identidade que pretendem projetar ou comunicar (WITT, 2010). Nesse sentido, o consumo do sistema operacional Ubuntu pode contribuir para a valorização de uma pessoa perante a comunidade de usuários. Além disso, contribui para alterar o status social dos usuários ou até mesmo comprometer sua reputação (DEANS, THOMAS, DAUBE et al., 2016). Portanto, a atenção ao escolher tal software torna-se extremamente importante para as relações sociais dos indivíduos, passando a integrar seu cotidiano.

Shang, Chen e Huang (2012) usaram as teorias do consumo simbólico, representação do self e ambiente mediado por computador para investigar por que as pessoas compram bens simbólicos decorativos no mundo virtual para enfeitar avatares. Eles mostram que as pessoas acessam esses elementos simbólicos virtuais para levar em consideração seus valores e pressões sociais e emocionais. Em meio a suas interações sociais, esses usuários compram bens virtuais para agradar a eles próprios e fazer amigos mediante a projeção de uma imagem desejável, a fim de obter respostas emocionais positivas dos outros.

A assunção da migração para o Ubuntu faz com que ele se torne parte da identidade do indivíduo que o adotou, o que nos conduz a uma discussão de múltiplos selves e o potencial de um indivíduo ser mudado por meio do consumo (GECAS, 1982), da mesma forma que a relação entre pertencer a um grupo particular e consumo (FISHER, BOLAND JUNIOR e LYYTINEN, 2016). Em nosso estudo, descobrimos que o comportamento dos usuários do Ubuntu foi expresso através de diversos selves (amigáveis, educados, ajudantes), que se adaptam a diferentes situações sociais nas quais eles operam. Os usuários estabelecem sua representação por meio desses selves.

Da mesma forma, Min e Lee (2011) investigaram a representação virtual do self de 312 blogueiros e observaram o perfil dos usuários como indivíduos heterogêneos, que revelam seus selves que vão se transformando de acordo com a dinâmica existente entre o usuário, a tecnologia e as tarefas a serem executadas em diferentes contextos sociais de uso. Isso ocorre ao longo do processo de documentação de sua vida cotidiana, onde expressam emoções, articulam ideias e opiniões. Assim, a blogosfera é um mediador e um rico espaço de controle a partir do qual os usuários se apresentam para si mesmos e para a alteridade (GOFFMAN, 1990), através de seus posts que refletem quem são e o que pensam, gostam e têm. Deve-se notar que no ciberespaço há a vantagem de se escolher em quais aspectos do self focar, como demonstrado nos estudos de Behm-Morawitz (2013).

No exemplo a seguir, há uma declaração sobre "uma declaração de amor: Eu amo o projeto Ubuntu!", no qual em determinado momento um dos interagentes afirma: "O Ubuntu mudou minha vida! Mudou minha maneira de ver o mundo, mudou minha ideologia! Ideologiaaaaa eu tenho um para me ajudar a viver!" (D14T2C1).

Este extrato deixa claro que um dos interagentes parou de usar o sistema operacional Windows e adotou o Ubuntu e, portanto, precisa declarar que faz parte da comunidade de usuários, formada por pessoas que compartilham a mesma ideologia 
Consumo simbólico e representação do self: um estudo de interações em uma comunidade virtual de usuários Ubuntu-Br
Osíris Luís da Cunha Fernandes | Nelson da Cruz Monteiro Fernandes Fernando Gomes de Paiva Júnior | André Luiz Maranhão de Souza Leão Marconi Freitas da Costa

(humanidade com os outros). Este é um significado embutido no sistema operacional Ubuntu ao qual o usuário quer se associar, e é bastante notável que a adesão das pessoas ao Ubuntu e todos os valores e princípios associados a ele, sejam adotadas em suas próprias vidas. A declaração de migração para o Ubuntu é demonstrada pelo uso de aspectos paralinguísticos, com utilização de '!' (entoação) no final das frases, e 'ideologiaaaaa' (duração do enunciado) para enaltecer os valores simbólicos (SCHOUTEN, 1991) associado ao Ubuntu e corroborar a assunção desses valores por outros membros da comunidade.

Na mesma declaração, os interagentes fazem um apelo aos apoiadores:

“Não esquece de dar suporte técnico às pessoas, caso elas tenham algum problema. Ajude-as, vá até a casa delas se for necessário, não adianta largá-las no sistema e dizer "se vira". Você tem que ajudar a pessoa a aprender o sistema, ou então, corre o risco de ela voltar pro Windows e ficar com uma má impressão formada sobre Linux (ao invés de boa)" (D14T2C1).

Neste caso, o uso de aspectos da definição de "eu" (footing) com o uso de aspas em "se vira" e os parênteses em "ao invés de boa ", mostra a definição de comportamento adequado (ROBINSON, 2007) de acordo com usuários do Ubuntu. Eles se preocupam com o compartilhamento de conhecimento, ao contrário do que acontece com os usuários do Windows, deixam uns aos outros sozinhos para resolverem seus problemas de forma individual.

Além disso, ao afirmar "tem que ajudar a pessoa a aprender sobre o sistema, ou então, há o risco de a pessoa voltar pro Windows e ficar com uma má impressão formada sobre o Linux (ao invés de boa)]" o interagente corrobora a ideia de que a migração para o Ubuntu requer suporte técnico e ajuda de usuários mais experientes. Assim, ao declarar a migração para o Ubuntu, oferece-se para dar suporte no fórum da comunidade dos usuários. Além disso, esse usuário demonstrou preocupação em proteger os usuários do Ubuntu, preservando sua característica de identidade como colaborador, comprometido com a partilha de conhecimentos (KEDZIOR, ALLEN e SCHROEDER, 2016).

O próximo extrato apresenta uma situação de interação entre iniciantes no Ubuntu, em que um dos interagentes acaba de instalar o sistema operacional Ubuntu e diz: "ps: uso o Ubuntu há 40 minutos e muito show (expressão facial - sorrindo)". Aqui, o uso de aspectos extralinguísticos (expressão facial: interpretado como um grande sorriso) aspectos paralinguísticos (variação ortoépica: ps:) e aspectos da definição do "eu" (alternância de código: 40 minutos; manifesta) são usados para enaltecer a declaração de migração para o Ubuntu e demonstrar satisfação pela imersão do novo usuário/interagente em uma nova comunidade identitária. Assim, o valor simbólico do Ubuntu serve como uma nova oportunidade para a representação de certos valores sociais, ligados a questões de aceitação social, conforme defendido por Killoran (2012).

\section{A expressão de sentimentos ao Ubuntu como forma de representação do Self}

A representação do self nas interações virtuais também ocorre por meio da expressão de sentimentos devotados (GRUBB e GRATHWOHL, 1967) ao Ubuntu. Nestas situações, os aspectos paralinguístico, extralinguístico e de definição do "eu" são utilizados para caracterizar e enfatizar as características do Ubuntu, bem como para corroborar e preservar os sentimentos dos usuários em relação a esse sistema operacional. Os extratos a seguir são elucidativos do uso desses aspectos. Na primeira situação de interação, um membro da comunidade afirma: “Eu te amo Ubuntu! Só uma coisa: pode sair distribuindo CDs do Ubuntu, Kubuntu, Xubuntu por aí" (D72T2C1).

O uso de exclamação ('!') depois de "Eu te amo Ubuntu" denota a utilização de aspectos paralinguísticos (entoação) para enfatizar um aspecto simbólico do consumo do Ubuntu, expresso em forma de amor. Assim, o uso de aspectos da definição do "eu" é evidenciado na referência às várias distribuições do Sistema Operacional 'Ubuntu, Kubuntu, Xubunt' (conhecimento de mundo nesta situação) e a forma de instalação mais utilizada, via "CD" (alternância de código nesta situação). Esses aspectos são usados para enfatizar tanto a diversidade das distribuições do Ubuntu, como para suportar a sensação da qualidade do sistema operacional e, por isso, o interagente recomenda fortemente, "pode sair distribuindo CDs". As várias versões do Ubuntu são nomeadas e servem como mecanismos para a expressão de uma identidade, através do qual ele nutre um sentimento de amor. Além disso, também mostra que ele está ciente do que acontece na comunidade e tem dominado seus códigos linguísticos.

Na situação de interação seguinte, outro interagente declara: “Estou tendo um caso com o Ubuntu também... minha namo não pode saber disto ... (expressão facial: P: P" (D72T1C4). 
Consumo simbólico e representação do self: um estudo de interações em uma comunidade virtual de usuários Ubuntu-Br
Osíris Luís da Cunha Fernandes | Nelson da Cruz Monteiro Fernandes Fernando Gomes de Paiva Júnior | André Luiz Maranhão de Souza Leão Marconi Freitas da Costa

Neste caso, os aspectos extralinguísticos (expressão facial: P: P, sorriso seguido de riso) e aspectos paralinguísticos (duração do enunciado: “...") são utilizados para corroborar o sentimento nutrido pelo Ubuntu (uma paixão oculta). Além disso, o usuário adota uma metáfora "Tenho um caso com o Ubuntu ..." para enfatizar que além da "garota" (variação ortoépica, usada em vez de namorada) ele tem outra paixão, o Ubuntu. Ao dizer "minha namo não pode saber disto ..." o interagente revela uma preocupação em preservar o seu self (face) para que não seja reconhecido como um traidor na comunidade. Por outro lado, o uso da expressão facial ao final dessa frase, concebida como sorriso malicioso seguido de risada, revelam certa ironia, ao mesmo tempo em que enfatiza a metáfora do ente amoroso.

Portanto, é possível dizer que o Ubuntu também é consumido como um sistema ideal para a busca de experiências vivas e múltiplas, capazes de preencher o espaço entre o interior e o exterior, entre o mundo pessoal e público dos usuários (SHANG, CHEN e HUANG, 2012). Assim, os consumidores criam múltiplas identidades para esse software, com as quais eles se protegem, enquanto internalizam seus significados e valores (LUNA-CORTES, 2017), tornando-os parte de si mesmos.

A expressão dos sentimentos com respeito à web representa uma consequência natural das experiências do consumidor no mundo virtual, uma vez que esse mundo se torna parte do que Belk (2013) chama de extensão do self. Consequentemente, os sentimentos desenvolvidos dentro deste universo virtual tornam-se parte da rica coleção de experiências que refletem a ideia de quem somos e o que pensamos (CASALO, FLAVIAN e GUINALIU, 2010). Quando os outros reconhecem esses sentimentos na comunidade, reforçam sua inserção na rede e desenvolvem interações com significados positivos (FISHER, BOLAND JUNIOR e LYYTINEN, 2016). Isso contribui, segundo Solomon (1983), para o alinhamento de seus sentimentos subjetivos com lugares objetivos que ocupam no mundo social e cultural.

\section{O suporte no fórum da comunidade virtual como um espaço de representação do self}

Fornecer suporte no fórum das comunidades Ubuntu, especialmente para iniciantes no uso do sistema operacional Ubuntu, é uma das formas de representação do self usadas nas interações virtuais. Nesse sentido, os aspectos paralinguísticos (GUMPERZ, 1982) e de definição do "eu" são utilizados para chamar a atenção, enfatizar, enaltecer e sugerir a necessidade de suporte no fórum da comunidade.

A situação de interação abaixo mostra a resposta de um membro da comunidade à pergunta de um iniciante no uso do Ubuntu sobre como proceder no fórum em caso de dúvidas relacionadas ao uso do software. Ele diz, "tenta acessar o canal \#ubuntu-br que tem bastante conteúdo e suporte imediato! "A gente nunca para!" (D15T3C15).

A demonstração de conhecimento da comunidade é visível, uma vez que o respondente recomenda o "canal \#ubuntu-br". O uso de exclamação ao final da frase "tenta acessar o canal \#ubuntu-br que tem muito conteúdo e suporte imediato!" representa uma entoação (aspecto paralinguístico) (SAVILLE-TROIKE, 2003), dada ao discurso do respondente para chamar a atenção para si e para a comunidade, a fim de mostrar que a comunidade se preocupa em compartilhar conhecimento e dar suporte técnico aos seus membros, fato que merece ser destacado.

Por outro lado, a entoação também tem a função de enfatizar a capacidade de resposta do suporte "imediato" e deixar claro que os usuários do Ubuntu sempre encontrarão suporte/ajuda no fórum de sua comunidade. Por fim, o usuário escreve: "A gente nunca para!" onde também encontramos o uso de aspectos paralinguísticos (entoação: '!') e aspectos da definição de "eu" (footing, pelo uso de aspas) para sugerir que o suporte no fórum é constante. Essa resposta também soa como uma tentativa de salvamento da face, provavelmente para preservar possíveis questionamentos sobre atrasos ou falhas em responder às dúvidas dos membros da comunidade (GUAN, WANG, JIN et al., 2018).

Os indivíduos procuram comprar objetos que transmitem seu estilo de vida (LANIER JUNIOR e RADER, 2017) e deixem claro para seus pares sua posição social e sua forma de agir e ver o mundo. Assim sendo, o uso do Ubuntu pode indicar que a pessoa é moderna, jovem e conhecedora dos avanços no campo das tecnologias de informação e comunicação (TIC). Além disso, o significado desse software decorre de sua capacidade de atuar como um indicador de status social (WITT, 2010). Assim, o consumo simbólico do Ubuntu é uma prática que impulsiona a relação entre seus usuários.

Um resultado semelhante aparece no estudo de Manago, Graham, Greenfield et al. (2008), que explora as formas pelas quais os jovens experimentam redes sociais dentro do MySpace no desenvolvimento de identidades pessoais, sociais e de gênero. Os autores identificaram que as relações peer-to-peer entre os estudantes sugerem que eles usam o MySpace para explorar identidades, se compararem socialmente e expressarem aspectos idealizados do self com os quais eles se identificam. 
Consumo simbólico e representação do self: um estudo de interações em uma comunidade virtual de usuários Ubuntu-Br
Osíris Luís da Cunha Fernandes | Nelson da Cruz Monteiro Fernandes Fernando Gomes de Paiva Júnior | André Luiz Maranhão de Souza Leão Marconi Freitas da Costa

Nesse sentido, os sites de redes sociais oferecem boas oportunidades para que os jovens percebam seus vários selves possíveis (LUNA-CORTES, 2017). Essas comunidades estão enfrentando novas ferramentas culturais para a construção de identidades em suas interações cotidianas. Logo, isso mostra que os usuários do sistema operacional estudado transformam ideias sobre o self em imagens objetivadas e tais imagens visam o alcance de aprovação pública por parte de suas audiências.

Em outra situação de interação, outro membro da comunidade comenta sobre o comprometimento de um colega da comunidade ao afirmar: "O cara nem doente, e "costurado" consegue ficar longe, hehehehe. Esse "Credo" (brincando com as palavras "Credo", que é o nome do usuário que está doente e "Credo" uma expressão equivalente a "oh gosh") ..." (D19T2C7).

Nessa situação, o uso de aspectos extralinguísticos e paralinguísticos (ERVIN-TRIPP, 1972) visa chamar a atenção para um membro da comunidade que mesmo doente não conseguiu se afastar do fórum da comunidade. "O cara" representado por uma alternância de código usada para elogiar o compromisso de uma pessoa com o apoio no fórum, também serve para enfatizar sua preocupação com os colegas da comunidade. "Costurado" entre aspas representa o aspecto footing da interação numa tentativa de mostrar um posicionamento diferenciado da pessoa em questão que mesmo operada não esquece as demais. Este elemento mostra um componente simbólico associado ao uso do Ubuntu, a humanidade com o outro, mostrando que os consumidores/usuários do Ubuntu podem se sacrificar para ajudar o próximo em uma situação difícil.

A expressão facial (hehehehe), concebida como gargalhada, foi usada para enaltecer a atitude do usuário por meio de uma demonstração de alegria/contentamento. Aqui vemos um indivíduo cujo self está alinhado com o conceito de autoimagem de Goffman, segundo o qual o humano é um ser socialmente construído pela alteridade (GOFFMAN, 1990), ou seja, a identidade do "cara" que "[...] nem doente e 'costurado' consegue ficar longe[...]". Isso pressupõe desse papel social do desenvolvedor, cujo principal objetivo é conhecer a si mesmo e aos outros.

Finalmente, como sinalizou Certeau (1984), as pessoas podem criar novos significados para situações sociais previamente estabelecidas (chamadas pelo autor de "artes de fazer"), e a carga simbólica que os produtos carregam pode ser incluída nesse campo. Assim, por essa lógica, as funções sociais dos produtos tornam-se centrais, sendo esse deslocamento de significação criado pela apropriação do que cada indivíduo faz e que, quando reconhecida pelos pares, movimenta as engrenagens das relações sociais (NOBLE e WALKER, 1997). Portanto, decisões, intenções e práticas no campo do self influenciam a vida social geral e são influenciadas por ela, num movimento interativo e capaz de construir a vida humana (HILSEN e HELVIK, 2014).

\section{CONCLUSÃo}

Este estudo adotou a sociolinguística interacional (GOFFMAN, 1967) como lente teórica e a netnografia da comunicação (KEATING, 2001) como método por congregar a natureza funcional da análise do discurso realizada. Essa perspectiva enfoca os aspectos culturais usados durante a interação das pessoas (verbal e não verbal) dentro de uma comunidade.

Escolhemos a abordagem funcional da análise do discurso devido à nossa busca por compreender a representação do self em interações no âmbito das comunidades virtuais através do consumo simbólico do software livre Ubuntu. Usamos tanto a concepção de Self do Goffman quanto a de consumo simbólico de Ravasi e Rindova (2008), a fim de contribuir para uma visão alternativa àquelas apresentadas nas pesquisas atuais no campo dos estudos organizacionais.

As pessoas adaptam suas formas de comunicação à Internet e se esforçam para expressar os aspectos não-verbais mais difíceis de serem destacados do contexto em interações face-a-face, dados seus fatores culturais e sociolinguísticos inerentes que ultrapassam os limites da voz. Assim, para a análise do corpus formado com o conjunto dos dados coletados na comunidade virtual Ubuntu do Brasil, utilizamos o protocolo de análise de dados desenvolvido por Freitas e Leão (2012) como meio de acessar e interpretar as linguagens verbais e não-verbais utilizadas em interações nesta comunidade virtual. Após a netnografia, a questão de pesquisa: como ocorre a representação do self em interações virtuais através do consumo simbólico do Sistema Operacional Ubuntu? foi abordada.

Em sua produção simbólica, os desenvolvedores vinculam ao sistema operacional Ubuntu princípios e valores culturais como a liberdade (mudar o código fonte, adaptação do software às necessidades individuais), o compromisso com o compartilhamento de conhecimentos, principalmente através de suporte técnico no fórum da comunidade identitária dos membros e a noção de humanidade entre si. Assim, podemos inferir que os princípios do Ubuntu são utilizados na dinâmica de representação do self de seus usuários, e fazem parte de sua socialização nas comunidades identitárias. 
Consumo simbólico e representação do self: um estudo de interações em uma comunidade virtual de usuários Ubuntu-Br
Osíris Luís da Cunha Fernandes | Nelson da Cruz Monteiro Fernandes Fernando Gomes de Paiva Júnior | André Luiz Maranhão de Souza Leão Marconi Freitas da Costa

Em interações virtuais, o Ubuntu é usado como um signo com significados compartilhados que funciona na representação do self dos usuários do Ubuntu na comunidade virtual. Assim, o estilo de vida baseado na ideologia Ubuntu foi intensamente sinalizado nas interações observadas. Em muitas situações, os aspectos simbólicos apareciam, por exemplo, através do uso de aspectos paralinguísticos como a entoação, seja por meio do uso da afirmação como da exclamação, enaltecendo a migração de usuários do Windows para o Ubuntu, ou seja, a adoção de um estilo de vida baseado nos princípios do Ubuntu. Nessa perspectiva, a percepção do valor do uso do Ubuntu está intimamente relacionada aos elementos simbólicos associados a ele, como o compromisso de compartilhar conhecimento e humanidade com os outros.

Além disso, notamos que quando o consumo é representativo do self, os usuários do Ubuntu utilizam os recursos simbólicos associados ao sistema operacional para se projetarem em sua comunidade identitária, Ubuntu-Br, como pessoas felizes, amigáveis, modernas e conhecedoras do mundo do software livre. Portanto, o Ubuntu é consumido mais dependendo dos significados incorporados nele durante sua produção, o que o torna um excelente signo para as interações com o potencial de permitir a caracterização de uma identidade, uma cultura (o self). Verificou-se também que, em diferentes momentos, o consumo simbólico deste software esteve associado à compreensão compartilhada dos usuários sobre o modo de agir na comunidade Ubuntu-Br, caracterizando o compartilhamento de ideais, valores e princípios como fatores preponderantes de admissão e permanência na comunidade virtual Ubuntu-Br.

O estudo corrobora a hipótese de que, no consumo de sistemas operacionais de código aberto, a propriedade sobre a customização é utilizada para a representação do self em comunidades virtuais. Como principais conclusões deste estudo, identificamos três formas primordiais de representação do self nas interações virtuais através do consumo simbólico do software livre Ubuntu: 1) Declaração de migração do Windows para o Ubuntu - os recursos simbólicos atrelados ao sistema operacional de código aberto permitem que a declaração de migração do sistema operacional Windows para Ubuntu seja utilizada como uma forma de autorepresentação na comunidade Ubuntu-Br por meio do uso de aspectos paralinguísticos e aspectos da definição do "eu", visando projetar, corroborar e elogiar os valores atribuídos ao Ubuntu e ao reconhecimento de novos membros na comunidade identitária dos usuários deste software; 2) Expressão de sentimentos para o Ubuntu principalmente através do uso de aspectos extralinguístico e da definição do "eu" para caracterizar e enfatizar as características do Ubuntu, bem como para apoiar e preservar os sentimentos dos usuários por este software; 3) Prestar suporte técnico no fórum da comunidade virtual - através do uso de aspectos paralinguísticos e aspectos da definição do "eu", para chamar a atenção, enfatizar, elogiar e sugerir a prestação de suporte técnico no fórum por um dos membros da comunidade.

Finalmente, essas representações do self permitem aos usuários de software livre a projeção de uma autoimagem desejada nas interações em comunidades virtuais. As formas de representação do self inferidas neste estudo não esgotam todas as possibilidades. Em vez disso, abrem espaço para um uso gerencial, como uma nova opção para a produção simbólica de software (livre e proprietário), considerando a capacidade dos componentes simbólicos atrelados a eles se tornarem parte da identidade/self dos usuários. Assim, uma das implicações que podemos extrair deste estudo é que o consumo autorepresentativo de sistemas operacionais de código aberto desempenham um papel fundamental na construção do self em termos que vão além do consumo de bens materiais no mundo offline, especialmente porque os recursos simbólicos associados ao software livre são utilizados como elementos de diferenciação dos usuários de sistemas proprietários. 
Consumo simbólico e representação do self: um estudo de interações em uma comunidade virtual de usuários Ubuntu-Br
Osíris Luís da Cunha Fernandes | Nelson da Cruz Monteiro Fernandes Fernando Gomes de Paiva Júnior | André Luiz Maranhão de Souza Leão Marconi Freitas da Costa

\section{REFERÊNCIAS}

ALMEIDA, S. O. et al. Efeitos da comunidade de origem no participante de comunidades virtuais de marca. RAE-Revista de Administração de Empresas, v. 52, n. 2, p. 204-216, 2012.

ALVESSON, M.; SKOLDBERG, K. Reflexive methodology: new vistas for qualitative research. 2. ed. London: SAGE Publications, 2009.

BAUMHAMMER, P.; SILVA, M. G.; COSTA, M. F. Aspectos simbólicos do smartphone e o eu estendido: um estudo do comportamento do consumidor português. Revista Interdisciplinar de Marketing, v. 7. n. 2, p. 175-190, 2017.

BELK, R. W. Extended self in a digital world. Journal of Consumer Research, v. 40, n. 3, p. 477-500, 2013.

BEHM-MORAWITZ, E. Mirrored selves: The influence of self-presence in a virtual world on health, appearance, and well-being. Computers in Human Behavior, v. 29, n. 1, p. 119-128, 2013.

BLUMER, H. Symbolic interactionism: perspective and method. 6. ed. Berkeley: California University Press, 1986.

BRINK, M. A. Rijkswaterstaat on the horns of a dilemma. Delft: Eburon, 2009.

BOWLER, G. M. Netnography: A method specifically designed to study cultures and communities online. The Qualitative Report, v. 15, n. 5, p. 1270-1275, 2010.

CASALO, L. V.; FLAVIAN, C.; GUINALIU, M. Relationshipquality, communitypromotion and brandloyalty in virtual communities: evidencefromfree software communities. International Journal of Information Management, v. 39, n. 4, p. 357-367, 2010.

CASTELLS, M. The internet galaxy: Reflections on the internet, business, and society. Oxford: Oxford University Press, 2002.

CASTELLS, M. The rise of the network society. 2. ed. West Sussex: The John Wiley \& Sons, 2010.

CERTEAU, M. The practice of every Day life. Berkeley: University of California Press, 1984.

CHANG, K. W.; LI, S. Y. Understanding consumer-to-consumer interactions in virtual communities: the salience of reciprocity. Journal of Business Research, v. 63, n. 9, p. 1033-1040, 2010.

COMOR, E. Contextualizing and critiquing the fantastic prosumer Power, alienation and hegemony. Critical Sociology, v. 37, n. 3, 2010. 309-327 p.

DEANS, E., et al. Creating symbolic cultures of consumption: ananalys is of the content of sports wagering advertisements in Australia. BMC Public Health, v. 16, n. 208, p. 1-11, 2016.

DIONYSIOU, D. D.; TSOUKAS. H. Understandingthe (re)creation of routines from within: A symbolic interactionist perspective. Academy of Management Review, v. 38, n. 2, p. 181-205, 2013.

EKINCI, Y.; SIRAKAYA-TURK, E.; PRECIADO, S. Symbolic consumption of tourism destination brands. Journal of Business Research, v. 66, n. 6 , p. 711-718, 2013.

ENGWARD, H.; DAVIS, G. Being reflexive in qualitative grounded theory: discussion and application of a model of reflexivity. Journal of Advanced Nursing, v. 71, n. 7, p. 1530-1538, 2015.
ERVIN-TRIPP, S. On sociolinguistic rules: Alternation and co-occurrence. In: GUMPERZ, J.; HYMES, D. (Eds.). Directions in sociolinguistics: the ethnography of communication. Basil Blackwell: New York, 1972.

FARAH, I. Ethnographyof communication. In: HORNBERGER, N. H.; CORSON, D. (Eds.). Encyclopedia of language and education. Dordrecht: Springer, 1997.

FISHER, M.; BOLAND JUNIOR., R.; LYYTINEN, K. Social networking as theproduction and consumption of a self. Information and Organization, v. 26, n. 4, p. 131-145, 2016.

FONSECA, M. J. et al. Tendências sobre as comunidades virtuais da perspectiva dos prosumers. RAE Electrônica, São Paulo, v. 7, n. 2, jul./dez. 2008. Disponível em: <http://dx.doi.org/10.1590/S167656482008000200008>. Acesso em: 09 set. 2019.

FREITAS, G. K.; LEAO, A. L. M. de S. A elaboração da face em comunidades virtuais de marca: um estudo de caso sobre uma comunidade virtual de consumidores da Coca-Cola. Cadernos EBAPE.BR, Rio de Janeiro, v. 10, n. 1, p. 181-201, 2012.

GBADAMOSI, A. Brand personification and symbolic consumption among ethnic minority teenage consumers: an empirical study. Journal of Brand Management, v. 22, n. 9, p. 737-754, 2015.

GECAS, V. The self-concept. Annual Review of Sociology, v. 8, p. 1-33, 1982.

GOLDEN-BIDDLE, K.; LOCKE, K. Appealing work: An investigation of how ethnographic texts convince. Organization Science, v. 4, n. 4 p. 595-616, 1993.

GOFFMAN, E. Interaction ritual: essays on face-to-face behavior. New York: Anchor, 1967.

GOFFMAN, E. Frame analysis: an essay on the organization of experience. New York: Harper and Row, 1974.

GOFFMAN, E. The presentation of self in evey Day life. London: Penguin, 1990.

GRUBB, E. L.; GRATHWOHL, H. L. Consumer self-concept, symbolism and market behavior: a theoretical approach. Journal of Marketing, v. 31, n. 4, p. 22-27, 1967.

GUAN, T. et al. Knowledge contribution behavior in online Q\&A communities: an empirical investigation. Computers in Human Behavior, v. 81, p. 137-147, 2018.

GUMPERZ, J. Sociolinguistics and communication in small groups. In: PRIDE, J. B.; HOLMES, J. (Eds.). Sociolinguistics. Penguin: Harmondsworth, Middlesex, 1972.

GUMPERZ, J.; HYMES, D. Directions in sociolinguistics: the ethnography of communication. Basil Blackwell: New York, 1972.

GUMPERZ, J. J. Discourse strategies. Cambridge: Cambridge University Press, 1982.

GUMPERZ, J. J. On interactional sociolinguistic method. In: SARANGI, S.; ROBERT, C. (Eds.). Talk, work and institutional order: discourse in medical, mediation and management settings. New York: Mouton de Gruyter, 1999. 
Consumo simbólico e representação do self: um estudo de interações em uma comunidade virtual de usuários Ubuntu-Br
Osíris Luís da Cunha Fernandes | Nelson da Cruz Monteiro Fernandes Fernando Gomes de Paiva Júnior | André Luiz Maranhão de Souza Leão Marconi Freitas da Costa
HABERMAS, J. Pensamento pós-metafísico: estudos filosóficos. 2. ed. Rio de Janeiro: Tempo Brasileiro, 2002.

HABIBI, M. R.; LAROCHE, M.; RICHARD, M. O. Testing an extended model of consumer behavior in the context of social media-based brand communities. Computers in Human Behavior, v. 62, p. 292 302, 2016.

HAMARI, J.; KERONEN, L. Why do people buy virtual goods: a metaanalysis. Computers in Human Behavior, v. 71, p. 59-69, 2017.

HAMMERL, M. et al. Attribution of symbolic brand meaning: The interplay of consumers, brands and reference groups. Journal of Consumer Marketing, v. 33, n. 1, p. 32-40, 2016.

HARMON-KIZER, T. R. et al. When multiple identities compete: The role of centrality in self-brand connections. Journalof Consumer Behavior, v. 12, n. 6, p. 483-495, 2013.

HILSEN, A. I.; HELVIK, T. The construction of self in social medias, such as Facebook. Al \& Society, v. 29, n. 1, p. 3-10, 2014

HILL, B. M.; BACON, J. O livro oficial do Ubuntu. Porto Alegre: Bookman, 2008.

HIMANEN, P. The hacker ethic and the spirit of capitalism. New York: Random House, 2001.

JANSEN, B.J.; SPINK, A.; TAKSA, I. Handbook of research on web log analysis. Hersey: IGI Global, 2009.

KEATING, E. The ethnography of communication. In: ATKINSON, P. et al. (Eds.). Handbook of ethnography. London: SAGE, 2001.

KEDZIOR, R.; ALLEN, D. E.; SCHROEDER, J. The selfie phenomenon: consumer identities in the social media marketplace. European Journalof Marketing, v. 50, n. 9, p. 1767-1772, 2016.

KILLORAN, J. B. Is it "about us"? Self-representation of technical communication consultants, independent contractors, and companies on the web. Technical Communication, v. 59, n. 4, p. 267-285, 2012.

KLEIN, H. K.; MYERS, M. D. A set of principles for conducting and evaluating interpretive field studies in information systems. MIS Quarterly, v. 23, n. 1, p. 67-94, 1999.

KOTARBA, J. A. Symbolic interaction and applied social research: a focus on translational science. Symbolic Interaction, v. 37, n. 3 , p. 412-425, 2014.

KOZINETS, R. V. The field behind the screen: Using netnography for marketing research in online communities. Journal of Marketing Research, v. 39, n. 1, p. 61-72, 2002.

LANIER JUNIOR, C. D.; RADER, C. S. Deconstructing symbolic consumption: exploring the anti-synthetic space between meaning and meaninglessness. Consumption Markets \& Culture, v.20, n. 3 , p. 215-244, 2017.

LESSIG, L. Cultura Livre: como a grande mídia usa a tecnologia e a lei para bloquear a cultura e controlar a criatividade. São Paulo: Trama, 2005.

LINCOLN, Y. S.; GUBA, E. G. Naturalistic inquiry. London: SAGE Publications, 1985.

LOWE, R.; POW, N.; SERBAN, I. V.; CHARLIN, L.; LIU, C. W.; PINEAU, $J$. Training end-to-end dialogue systems with the Ubuntu dialogue corpus. Dialogue \& Discourse, v. 8, n. 1, p. 31-65, 2017.
LUNA-CORTES, G. The influence of symbolic consumption on experience value and the use of virtual social networks. Spanish Journal of Marketing - ESIC, v. 21, n. 1, p. 39-51, 2017.

MANAGO, A. M. et al. Self-presentation and gender on MySpace. Journal of Applied Developmental Psychology, v. 29, n. 6, p. 446-458, 2008. Disponível em: <https://doi.org/10.1016/j.appdev.2008.07.001>. Acesso em: 09 set. 2019.

MEAD, G. H. Mind, self, and society. Chicago: The University of Chicago Press, 2015

McCAHILL, M.The surveillance web: the rise of visual surveillance in an English city. Cullompton: Willan Pub, 2002.

MCCRACKEN, G. Culture and consumption: new approaches to the symbolic character of consumer goods and activities. Bloomington: Indiana University Press, 1990.

MIN, J.; LEE, H. The change in user and IT dynamics: blogs as IT-enabled virtual self-presentation. Computers in Human Behavior, v. 27, n. 6, p. 2339-2351, 2011.

MORGAN, G. Transnational communities and business systems. Global Networks, v. 1, n. 2, p. 113-130, 2011.

MUELLER, F. Taking Goffman seriously: developing strategy-aspractice. Critical Perspectives on Accounting, v. 53, p. 16-30, 2018.

NOBLE, C. H.; WALKER, B. A. Exploring the relationship among liminal transitions, symbolic consumption, and the extended self. Psychology \& Marketing, v. 14, n. 1, p. 29-47, 1997.

PAIVA JUNIOR, F. G.; LEAO, A. L. M. S.; MELLO, S. C. B. Validade e confiabilidade na pesquisa qualitativa em Administração. Revista de Ciências da Administração, v. 13, n. 31, p. 190-209, 2011.

POLUKHINA, E.; STRELNIKOVA, A. Exploring the Russian online giftexchange communities: the results of nethnographic approach. The Qualitative Report, v. 20, n. 12, p. 2041-2049, 2015.

POZZEBON, M. Conducting and evaluating critical interpretive research: Examining criteria as a key component in building a research tradition. In: KAPLAN, B. et al. (Eds.) Information systems research: relevant theory and informed practice. Springer, Boston: Springer, 2004.

PRONAY, S.; HETESI, E. Symbolic consumption in the case of brand comunications. Society and Economy, v. 38, n. 1, p. 87-102, 2016.

RAVASI, D. RINDOVA, V. Symbolic value creation. In: BARRY, D.; HANSEN, $\mathrm{H}$. (Eds.). The sage handbook of new approaches in management and organization. London: Sage, 2008.

RITZER, G.; JUGERSON, N. Production, consumption, prosumption: The nature of capitalism in the age of the digital 'prosumer'. Journal of Consumer Culture, v. 10, n. 1, p. 13-36, 2010.

RITZER, G. Prosumption: Evolution, revolution, or eternal return of the same? Journal of Consumer Culture, v. 14, n. 1, p. 3-25, 2014.

RITZER, G. Prosumer capitalism. The Sociological Quartely, v. 56, p. 413-445, 2015.

ROBINSON, L. The cyberself: the self-ing Project goes online, symbolic interaction in the digital age. New Media \& Society, v. 9, n. 1, p. 93-110, 2007.

SAVILLE-TROIKE, M. The ethnography of communication: an introduction. Malden: Blackwell Pub, 2003. 
Consumo simbólico e representação do self: um estudo de interações em uma comunidade virtual de usuários Ubuntu-Br
Osíris Luís da Cunha Fernandes | Nelson da Cruz Monteiro Fernandes Fernando Gomes de Paiva Júnior | André Luiz Maranhão de Souza Leão Marconi Freitas da Costa
SCHAU, H.; GILLY, M. We are what we post? Self-presentation in personal web space. Journal of Consumer Research, v. 30, p. 285404, 2003.

SCHOUTEN, J. M. Selves in transition: symbolic consumption in personal rites os passage and identity reconstruction. Journal of Consumer Research, v. 17, n. 4, p. 412-425, 1991.

SCHULTZE, U. A confessional account of an ethnography about knowledge work. MIS Quarterly, v. 24, n. 1, p. 3-41, 2000.

SHANG, R. A.; CHEN, Y. C.; HUANG, S. C. A private versus a public space: anonymity and buying decorative symbolic goods for avatars in a virtual world. Computers in Human Behavior, v. 28, n. 6, p. 2227-2235, 2012.

SILVEIRA, S. A. Software livre: a luta pela liberdade do conhecimento. São Paulo: Fundação Perseu Abramo, 2004.

SODERBERG, J. Hacking capitalism: The free and open source software movement. New York: Routledge, 2012.

SOLOMON, M. R. The role of products as social stimuli: a symbolic interactionism perspective. Journal of Consumer Research, v. 10, n. 3, p. 319-329, 1983.
STALLMAN, R. Free software, free society: selected essays of Richard M. Stallman. Boston: GNU Press, 2002.

TANGSUPWATTANA, W.; LIU, X. Symbolic consumption and Generation $Y$ consumers: evidence from Thailand. Asia Pacific Journalof Marketing and Logistics, v. 29, n. 5, p. 917-932, 2017.

TRAUTH, E. M.; JESSUP, L. M. Understanding computer-mediated discussions: Positivist and interpretive analyses of group support system use. MIS Quarterly, v. 24, n. 1, p. 43-79, 2000.

VAN DIJCK, J. The culture of connectivity: a critical history of social media. New York: Oxford University Press, 2013.

VELLOZO, H. S. Ubuntu e a Popularização do Software-Livre. In: Anais do Congresso Nacional Universidade, EAD e Software Livre. Belo Horizonte, 2012, Belo Horizonte. Anais... Belo Horizonte: UFMG, 2012.

WITT, U. Symbolic consumption and the social construction of product characteristics. Structural Change and Economic Dynamics, v. 21, n. 1, p. 17-25, 2010.

WITTGENSTEIN, L. Philosophische Untersuchungen. Frankfurt: Suhrkamp Verlag, 2003.

Osíris Luís da Cunha Fernandes

ORCID: https://orcid.org/0000-0003-4158-6995

Doutor em Administração pela Universidade Federal de Pernambuco (UFPE); Diretor de Marketing e Operações do Instituto de Tecnologia de Pernambuco (ITEP), Recife - PE, Brasil. E-mail: osiriscunha@gmail.com

Nelson da Cruz Monteiro Fernandes

ORCID: https://orcid.org/0000-0002-1662-9300

Doutor em Administração pela Universidade Federal de Pernambuco (UFPE); Professor Adjunto da Universidade Federal de Pernambuco (UFPE), Caruaru-PE, Brasil.E-mail: cruzfernandes55@gmail.com

Fernando Gomes de Paiva Júnior

ORCID: https://orcid.org/0000-0001-5715-3882

Doutor em Administração pela Universidade Federal de Minas Gerais (UFMG); Professor Adjunto da Universidade Federal de Pernambuco (UFPE), Recife-PE, Brasil. E-mail: fernando.paivajr@pq.cnpq.br

André Luiz Maranhão de Souza Leão

ORCID: https://orcid.org/0000-0002-7660-5845

Doutor em Administração pela Universidade Federal de Pernambuco (UFPE); Professor Associado da Universidade Federal de Pernambuco (UFPE), Recife - PE, Brasil. E-mail: aleao21@hotmail.com

Marconi Freitas da Costa

ORCID: https://orcid.org/0000-0001-9888-8359

Doutor em Administração pela Universidade de São Paulo (FEA USP); Professor Adjunto da Universidade Federal de Pernambuco (UFPE), Caruaru - PE, Brasil. E-mail: marconi.fcosta@ufpe.br 